\title{
Complex Wishart Distribution Based Analysis of Polarimetric Synthetic Aperture Radar Data
}

\author{
Allan A. Nielsen \\ Technical University of Denmark \\ Danish National Space Center \\ IMM, Building 321 \\ DK-2800 Lyngby, Denmark \\ Email: aa@space.dtu.dk
}

\author{
Henning Skriver \\ Technical University of Denmark \\ Danish National Space Center \\ Ørsted•DTU, Building 348 \\ DK-2800 Lyngby, Denmark \\ Email: hs@space.dtu.dk
}

\author{
Knut Conradsen \\ Technical University of Denmark \\ Informatics and Mathematical Modelling \\ IMM, Building 321 \\ DK-2800 Lyngby, Denmark \\ Email: kc@imm.dtu.dk
}

\begin{abstract}
Multi-look, polarimetric synthetic aperture radar (SAR) data are often worked with in the so-called covariance matrix representation. For each pixel this representation gives a $3 \times 3$ Hermitian, positive definite matrix which follows a complex Wishart distribution. Based on this distribution a test statistic for equality of two such matrices and an associated asymptotic probability for obtaining a smaller value of the test statistic are given and applied to change detection, edge detection and segmentation in polarimetric SAR data. In a case study EMISAR $L$-band data from 17 April 1998 and 20 May 1998 covering agricultural fields near Foulum, Denmark, are used. Soon the Japanese ALOS, the German TerraSAR-X and the Canadian RADARSAT-2 will acquire space-borne, polarimetric data making analysis based on these methods important.
\end{abstract}

\section{INTRODUCTION}

Due to its all-weather mapping capability and independence of, for instance, cloud cover, synthetic aperture radar (SAR) data hold a strong potential for e.g. change detection studies, edge detection and segmentation in remote sensing applications. It is well-known that different targets in different locations in a SAR image exhibit different backscatter characteristics. It is also well-known that the development of different targets (e.g. crops) over time causes changes in the backscatter. In the crop case the radar backscattering is sensitive to the dielectric properties of the vegetation and the soil, to the plant structure (i.e., the size, shape, and orientation distributions of the scatterers), to the surface roughness, and to the canopy structure (e.g. row direction and spacing, and cover fraction) [1], [2].

Based on the complex Wishart distribution of the so-called covariance matrix representation of multi-look polarimetric SAR image data this paper describes methods to perform change detection, edge detection and segmentation in polarimetric SAR data of agricultural fields.

The polarimetric SAR measures the amplitude and phase of backscattered signals in four combinations of the linear receive and transmit polarizations: $\mathrm{HH}, \mathrm{HV}, \mathrm{VH}$, and $\mathrm{VV}(\mathrm{H}$ for horizontal and $\mathrm{V}$ for vertical polarization, respectively). These signals form the complex scattering matrix which relates the scattered to the incident electric fields [3]. The inherent speckle in the SAR data can be reduced by spatial averaging at the expense of loss of spatial resolution. In this so-called multi-look case a more appropriate representation of the backscattered signal is the covariance matrix in which the average properties of a group of resolution cells can be expressed in a single matrix. The average covariance matrix is defined as [3]

$$
\langle\boldsymbol{C}\rangle=\left[\begin{array}{ccc}
\left\langle S_{h h} S_{h h}^{*}\right\rangle & \left\langle S_{h h} S_{h v}^{*}\right\rangle & \left\langle S_{h h} S_{v v}^{*}\right\rangle \\
\left\langle S_{h v} S_{h h}^{*}\right\rangle & \left\langle S_{h v} S_{h v}^{*}\right\rangle & \left\langle S_{h v} S_{v v}^{*}\right\rangle \\
\left\langle S_{v v} S_{h h}^{*}\right\rangle & \left\langle S_{v v} S_{h v}^{*}\right\rangle & \left\langle S_{v v} S_{v v}^{*}\right\rangle
\end{array}\right]
$$

where $\langle\cdot\rangle$ denotes ensemble averaging, * denotes complex conjugation, and $S_{r t}$ is the complex scattering amplitude for receive polarization $r$ and transmit polarization $t$ ( $r$ and $t$ are either $h$ for horizontal or $v$ for vertical). Reciprocity, which normally applies to natural targets, gives $S_{h v}=S_{v h}$ (in the backscattering direction using the backscattering alignment convention [3]) and results in the covariance matrix (1) with rank 3. $\langle\boldsymbol{C}\rangle$ follows a complex Wishart distribution.

In this paper a test statistic for equality of two complex covariance matrices and an associated asymptotic probability measure for obtaining a smaller value of the test statistic are given and applied to change detection, edge detection and segmentation in fully polarimetric SAR data. In [4] a change detection scheme based on canonical correlations analysis is applied to scalar EMISAR data, see also [5]-[7].

As opposed to existing methods that work on each polarization (HH, VV, HV) individually followed by some heuristic fusion step, this test statistic allows for the full polarimetric information simultaneously.

If used with $\mathrm{HH}, \mathrm{VV}$ or $\mathrm{HV}$ data only our test statistic reduces to the well-known test statistic for equality of the scale parameters in two gamma distributions.

For a more thorough description of the method, see [8]; see also [9], [10]. For the edge detection application, see [11]-[13]. For the segmentation application, see [13]-[15]. See also [16]. Other applications of the complex Wishart distribution to SAR data not described here include (multitemporal) classification [17] and speckle reduction [18].

\section{THEORY}

This section very briefly describes the complex normal and Wishart distributions, and the likelihood ratio test for equal- 
ity of two complex Wishart matrices. For a more thorough description, see [8] and references therein.

\section{A. The Complex Normal Distribution}

We say that a $p$-dimensional random complex vector $Z$ follows a complex multivariate normal distribution with mean $\mathbf{0}$ and dispersion matrix $\boldsymbol{\Sigma}$, i.e., $\boldsymbol{Z}=\left[Z_{1} \cdots Z_{p}\right]^{T} \in N_{C}(\mathbf{0}, \boldsymbol{\Sigma})$ if the frequency function is

$$
\begin{aligned}
f(\boldsymbol{z}) & =\frac{1}{\pi^{p}|\boldsymbol{\Sigma}|} \exp \left\{-\boldsymbol{z}^{* T} \boldsymbol{\Sigma}^{-1} \boldsymbol{z}\right\} \\
& =\frac{1}{\pi^{p}|\boldsymbol{\Sigma}|} \exp \left\{-\operatorname{tr}\left[\boldsymbol{\Sigma}^{-1} \boldsymbol{z} \boldsymbol{z}^{* T}\right]\right\}
\end{aligned}
$$

where $|\cdot|$ denotes the determinant, tr denotes the trace of a matrix, and ${ }^{* T}$ denotes complex conjugation $\left(^{*}\right)$ and transpose $\left({ }^{T}\right)$. The complex vector $\left[\begin{array}{lll}S_{h v} & S_{h h} & S_{v v}\end{array}\right]^{T}$ whose elements appear in Equation 1 follows this distribution.

\section{B. The Complex Wishart Distribution}

We say that a Hermitian, positive definite random $p \times p$ matrix $\boldsymbol{W}$ follows a complex Wishart distribution, i.e., $\boldsymbol{W} \in$ $W_{C}(p, n, \boldsymbol{\Sigma})$ if the frequency function is

$$
f(\boldsymbol{w})=\frac{1}{\Gamma_{p}(n)} \frac{1}{|\boldsymbol{\Sigma}|^{n}}|\boldsymbol{w}|^{n-p} \exp \left\{-\operatorname{tr}\left[\boldsymbol{\Sigma}^{-1} \boldsymbol{w}\right]\right\}
$$

where

$$
\Gamma_{p}(n)=\pi^{p(p-1) / 2} \prod_{j=1}^{p} \Gamma(n-j+1)
$$

The frequency function is defined for $\boldsymbol{w}$ positive definite.

If $\boldsymbol{Z}_{i} \in N_{C}(\mathbf{0}, \boldsymbol{\Sigma}), i=1, \ldots, n$ are independent $(n>p)$, then $\boldsymbol{W}=\sum_{i=1}^{n} \boldsymbol{Z}_{i} \boldsymbol{Z}_{i}^{* T} \in W_{C}(p, n, \boldsymbol{\Sigma})$. The complex matrix $\langle\boldsymbol{C}\rangle$ in Equation 1 follows this distribution.

If $\boldsymbol{X}$ and $\boldsymbol{Y}$ are independent and both follow complex Wishart distributions, $\boldsymbol{X} \in W_{C}(p, n, \boldsymbol{\Sigma})$ and $\boldsymbol{Y} \in$ $W_{C}(p, m, \boldsymbol{\Sigma})$, then their sum also follows a complex Wishart distribution, $\boldsymbol{S}=\boldsymbol{X}+\boldsymbol{Y} \in W_{C}(p, n+m, \boldsymbol{\Sigma})$.

\section{Test for Equality of Two Complex Wishart Matrices}

Let the independent $p \times p$ Hermitian, positive definite matrices $\boldsymbol{X}$ and $\boldsymbol{Y}$ be complex Wishart distributed, i.e., $\boldsymbol{X} \in$ $W_{C}\left(p, n, \boldsymbol{\Sigma}_{x}\right)$ with $\hat{\boldsymbol{\Sigma}}_{x}=\frac{1}{n} \boldsymbol{X}$ and $\boldsymbol{Y} \in W_{C}\left(p, m, \boldsymbol{\Sigma}_{y}\right)$ with $\hat{\boldsymbol{\Sigma}}_{y}=\frac{1}{m} \boldsymbol{Y}$. We consider the null hypothesis $H_{0}: \boldsymbol{\Sigma}_{x}=\boldsymbol{\Sigma}_{y}$ which states that the two matrices are equal against the alternative hypothesis $H_{1}: \boldsymbol{\Sigma}_{x} \neq \boldsymbol{\Sigma}_{y}$.

If $H_{0}$ is true (in statistical parlance: "under $H_{0}$ ") $\boldsymbol{S}=\boldsymbol{X}+$ $\boldsymbol{Y} \in W_{C}(p, n+m, \boldsymbol{\Sigma})$ with $\hat{\boldsymbol{\Sigma}}=\frac{1}{n+m} \boldsymbol{S}=\frac{1}{n+m}(\boldsymbol{X}+\boldsymbol{Y})$. The likelihood ratio test statistic becomes

$$
Q=\frac{L(\hat{\boldsymbol{\Sigma}})}{L_{x}\left(\hat{\boldsymbol{\Sigma}}_{x}\right) L_{y}\left(\hat{\boldsymbol{\Sigma}}_{y}\right)}
$$

Here

$$
L_{x}\left(\hat{\boldsymbol{\Sigma}}_{x}\right)=\frac{1}{\Gamma_{p}(n)}\left|\frac{1}{n} \boldsymbol{X}\right|^{-n}|\boldsymbol{X}|^{n-p} \exp \{-n \operatorname{tr} \boldsymbol{I}\}
$$

(and similarly for $L_{y}\left(\hat{\boldsymbol{\Sigma}}_{y}\right)$ ) where $\boldsymbol{I}$ is the identity matrix $(\operatorname{tr} \boldsymbol{I}=p)$. For the numerator of $Q$ we get

$$
\begin{aligned}
L(\hat{\boldsymbol{\Sigma}})= & \frac{1}{\Gamma_{p}(n) \Gamma_{p}(m)}\left|\frac{1}{n+m}(\boldsymbol{X}+\boldsymbol{Y})\right|^{-(n+m)} \times \\
& |\boldsymbol{X}|^{n-p}|\boldsymbol{Y}|^{m-p} \exp \{-(n+m) \operatorname{tr} \boldsymbol{I}\} .
\end{aligned}
$$

This leads to the desired likelihood ratio test statistic

$$
Q=\frac{(n+m)^{p(n+m)}}{n^{p n} m^{p m}} \frac{|\boldsymbol{X}|^{n}|\boldsymbol{Y}|^{m}}{|\boldsymbol{X}+\boldsymbol{Y}|^{n+m}} .
$$

If $n=m$ we get

$$
\ln Q=n(2 p \ln 2+\ln |\boldsymbol{X}|+\ln |\boldsymbol{Y}|-2 \ln |\boldsymbol{X}+\boldsymbol{Y}|) .
$$

An asymptotic expression for the probability of finding a smaller value of $-2 \rho \ln Q$ is

$$
\begin{aligned}
& P\{-2 \rho \ln Q \leq z\} \simeq P\left\{\chi^{2}\left(p^{2}\right) \leq z\right\}+ \\
& \omega_{2}\left[P\left\{\chi^{2}\left(p^{2}+4\right) \leq z\right\}-P\left\{\chi^{2}\left(p^{2}\right) \leq z\right\}\right]
\end{aligned}
$$

with

$$
\rho=1-\frac{2 p^{2}-1}{6 p}\left(\frac{1}{n}+\frac{1}{m}-\frac{1}{n+m}\right)
$$

and

$$
\omega_{2}=-\frac{p^{2}}{4}\left(1-\frac{1}{\rho}\right)^{2}+\frac{p^{2}\left(p^{2}-1\right)}{24 \rho^{2}}\left(\frac{1}{n^{2}}+\frac{1}{m^{2}}-\frac{1}{(n+m)^{2}}\right)
$$

For covariance matrix data $p=3$. For $\mathrm{HH}, \mathrm{HV}$ or VV data $p=1$. In the latter case $\boldsymbol{X}$ and $\boldsymbol{Y}$ are therefore scalars $X$ and $Y$, and $Q$ reduces to

$$
Q=\frac{(n+m)^{n+m}}{n^{n} m^{m}} \frac{X^{n} Y^{m}}{(X+Y)^{n+m}}
$$

which is equivalent to the well-known likelihood ratio test statistic for the equality of two gamma parameters [19], [20].

The elements in the covariance matrix containing co- and cross-polarized components often contain little information, and for randomly distributed targets with azimuthal symmetry, these elements are zero. It is important to notice, however, that if the sample covariance matrix is forced into azimuthal symmetry, the sample covariance matrix no longer follows the complex Wishart distribution [8].

\section{Azimuthal Symmetry}

By setting the elements in the covariance matrix $\langle C\rangle$ in Equation 1 containing co- and cross-polarized components to zero, and swapping first rows and then columns two and three, we obtain in the azimuthal symmetry case

$$
\begin{gathered}
{\left[\begin{array}{ccc}
\left\langle S_{h h} S_{h h}^{*}\right\rangle & \left\langle S_{h h} S_{v v}^{*}\right\rangle & 0 \\
\left\langle S_{v v} S_{h h}^{*}\right\rangle & \left\langle S_{v v} S_{v v}^{*}\right\rangle & 0 \\
0 & 0 & \left\langle S_{h v} S_{h v}^{*}\right\rangle
\end{array}\right]=} \\
{\left[\begin{array}{cc}
\boldsymbol{X}_{1} & 0 \\
0 & \boldsymbol{X}_{2}
\end{array}\right]=\boldsymbol{X}}
\end{gathered}
$$

where $\boldsymbol{X}_{1}$ is $p_{1} \times p_{1}$ (here $2 \times 2$ ) and $\boldsymbol{X}_{2}=\left\langle S_{h v} S_{h v}^{*}\right\rangle$ is $p_{2} \times p_{2}$ (here $1 \times 1$ ). This matrix is not Wishart distributed. We now consider $\boldsymbol{X}_{1} \in W_{C}\left(p_{1}, n, \boldsymbol{\Sigma}_{x 1}\right), \boldsymbol{X}_{2} \in W_{C}\left(p_{2}, n, \boldsymbol{\Sigma}_{x 2}\right)$, 


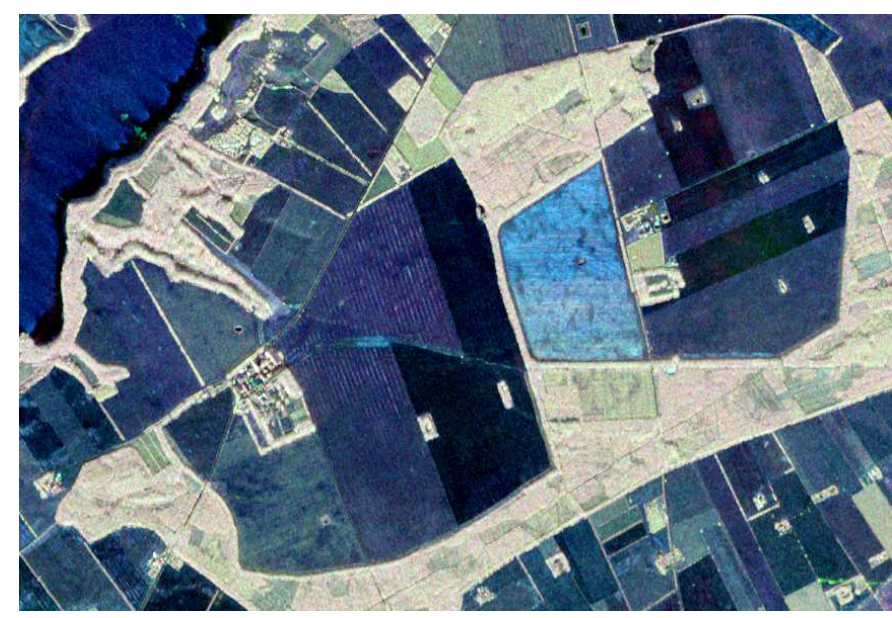

Fig. 1. $L$-band EMISAR data from 17 April 1998

$\boldsymbol{Y}_{1} \in W_{C}\left(p_{1}, m, \boldsymbol{\Sigma}_{y 1}\right), \boldsymbol{Y}_{2} \in W_{C}\left(p_{2}, m, \boldsymbol{\Sigma}_{y 2}\right)$, and we assume that $\boldsymbol{X}_{1}, \boldsymbol{X}_{2}, \boldsymbol{Y}_{1}$ and $\boldsymbol{Y}_{2}$ are mutually independent.

We want to test the hypothesis $H_{0}: \boldsymbol{\Sigma}_{x 1}=\boldsymbol{\Sigma}_{y 1}\left(=\boldsymbol{\Sigma}_{1}\right)$ and $\boldsymbol{\Sigma}_{x 2}=\boldsymbol{\Sigma}_{y 2}\left(=\boldsymbol{\Sigma}_{2}\right)$ against all alternatives. We have the likelihood function

$$
\begin{aligned}
& L\left(\boldsymbol{\Sigma}_{x 1}, \boldsymbol{\Sigma}_{x 2}, \boldsymbol{\Sigma}_{y 1}, \boldsymbol{\Sigma}_{y 2}\right)= \\
& \quad L^{(1)}\left(\boldsymbol{\Sigma}_{x 1}\right) L^{(2)}\left(\boldsymbol{\Sigma}_{x 2}\right) L^{(3)}\left(\boldsymbol{\Sigma}_{y 1}\right) L^{(4)}\left(\boldsymbol{\Sigma}_{y 2}\right) .
\end{aligned}
$$

The likelihood ratio test statistic becomes

$$
\begin{aligned}
Q & =\frac{(n+m)^{p(n+m)}}{n^{p n} m^{p m}} \frac{\left|\boldsymbol{X}_{1}\right|^{n}\left|\boldsymbol{Y}_{1}\right|^{m}\left|\boldsymbol{X}_{2}\right|^{n}\left|\boldsymbol{Y}_{2}\right|^{m}}{\left|\boldsymbol{X}_{1}+\boldsymbol{Y}_{1}\right|^{n+m}\left|\boldsymbol{X}_{2}+\boldsymbol{Y}_{2}\right|^{n+m}} \\
& =\frac{(n+m)^{p(n+m)}}{n^{p n} m^{p m}} \frac{|\boldsymbol{X}|^{n}|\boldsymbol{Y}|^{m}}{|\boldsymbol{X}+\boldsymbol{Y}|^{n+m}}
\end{aligned}
$$

where the latter equality is due to the fact that the determinant of a block diagonal matrix is the product of the determinants of the diagonal elements, i.e., we get the same test statistic as in the full covariance matrix case. In this case $p^{2}=p_{1}^{2}+p_{2}^{2}$. If

$$
\begin{aligned}
\rho & =\frac{1}{p^{2}}\left(p_{1}^{2} \rho_{1}+p_{2}^{2} \rho_{2}\right) \\
\rho_{i} & =1-\frac{2 p_{i}^{2}-1}{6 p_{i}}\left(\frac{1}{n}+\frac{1}{m}-\frac{1}{n+m}\right)
\end{aligned}
$$

and

$$
\begin{gathered}
\omega_{2}=-\frac{p^{2}}{4}\left(1-\frac{1}{\rho}\right)^{2}+\frac{p_{1}^{2}\left(p_{1}^{2}-1\right)+p_{2}^{2}\left(p_{2}^{2}-1\right)}{24} \times \\
\left(\frac{1}{n^{2}}+\frac{1}{m^{2}}-\frac{1}{(n+m)^{2}}\right) \frac{1}{\rho^{2}}
\end{gathered}
$$

then an asymptotic expression for the probability of finding a smaller value of $-2 \rho \ln Q$ is

$$
\begin{aligned}
& P\{-2 \rho \ln Q \leq z\} \simeq P\left\{\chi^{2}\left(p^{2}\right) \leq z\right\}+ \\
& \omega_{2}\left[P\left\{\chi^{2}\left(p^{2}+4\right) \leq z\right\}-P\left\{\chi^{2}\left(p^{2}\right) \leq z\right\}\right] .
\end{aligned}
$$

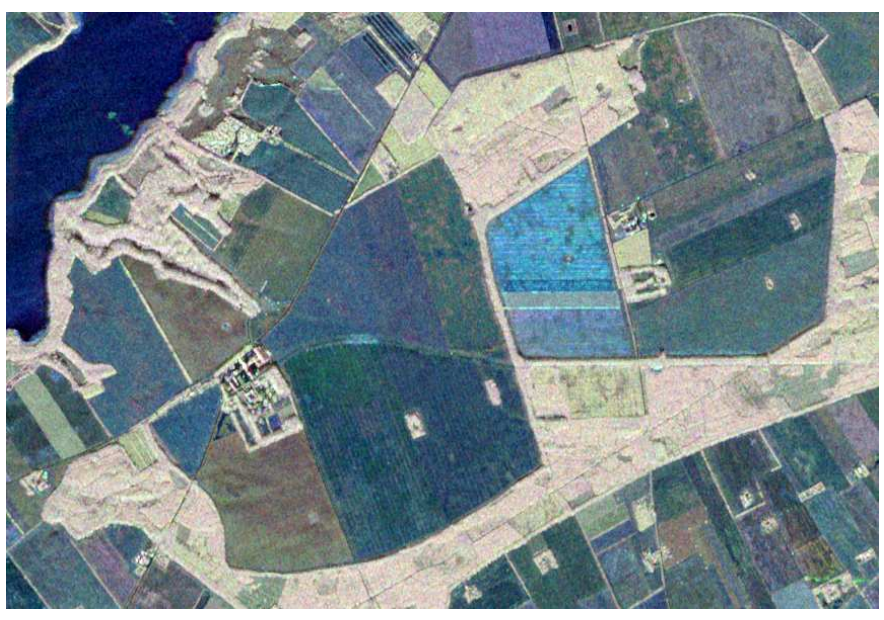

Fig. 2. $\quad$ L-band EMISAR data from 20 May 1998

\section{CASE STUdy: EMISAR DATA}

The EMISAR is the result of a research and development project initiated in 1986 at the Department of Electromagnetic Systems (now part of the Danish National Space Center) of the Technical University of Denmark [21], [22]. The EMISAR system is fully polarimetric and interferometric and it operates at two frequencies, $L$-band $(1.25 \mathrm{GHz} / 25 \mathrm{~cm}$ wavelength) and $C$-band (5.3 GHz/5.7 cm wavelength). The EMISAR is flown on a Royal Danish Airforce Gulfstream G-3 aircraft and is normally operated from an altitude of approximately 12,500 $\mathrm{m}$, the spatial resolution is $2 \times 2 \mathrm{~m}^{2}$ (one-look), the ground range swath is approximately $12 \mathrm{~km}$, and typical incidence angles range from $35^{\circ}$ to $60^{\circ}$. The processed data from this system are fully calibrated by means of an advanced internal calibration system.

To illustrate the change and edge detection, and segmentation capabilities of the derived test statistic this case study uses EMISAR data from an agricultural test site at the Research Center Foulum located in Central Jutland, Denmark. The data used here are part of the data used in a previously reported study on polarimetric signatures of crops [2].

Two EMISAR $L$-band images from 17 April 1998 and 20 May 1998 are shown in Figures 1 and 2 as color composites of the $\mathrm{HV}$ (actually the complex addition of $\mathrm{HV}$ and $\mathrm{VH}$; red), $\mathrm{HH}$ (green), and VV (blue) channels. The HH and VV channels are stretched linearly from $-30 \mathrm{~dB}$ to $0 \mathrm{~dB}$ and the $\mathrm{HV}$ channel is strethed from $-36 \mathrm{~dB}$ to $-6 \mathrm{~dB}$. The single look scattering matrix data have been converted to covariance matrix data, and at the same time speckle reduced by a raised cosine filter to an equivalent number of looks of approximately $11(=n=m)$.

The geometrical co-registration is very important in a change detection application where two images are compared on a pixel-by-pixel basis. The polarimetric images were registered to a digital elevation model generated from interferometric data acquired by EMISAR. The registration was carried out by combining a model of the imaging geometry with a few ground control points, and the images were registered to one 


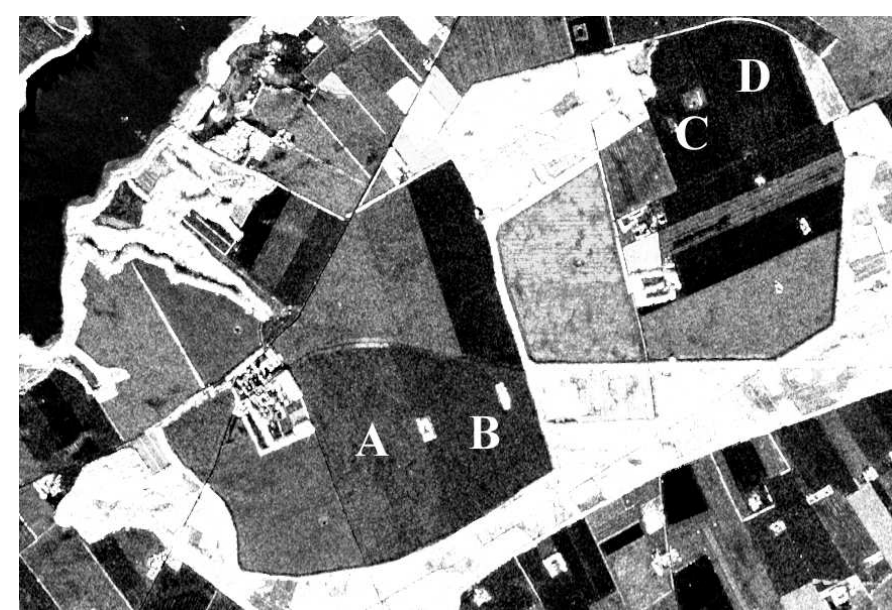

Fig. 3. $L$-band EMISAR data from 20 May 1998, span image

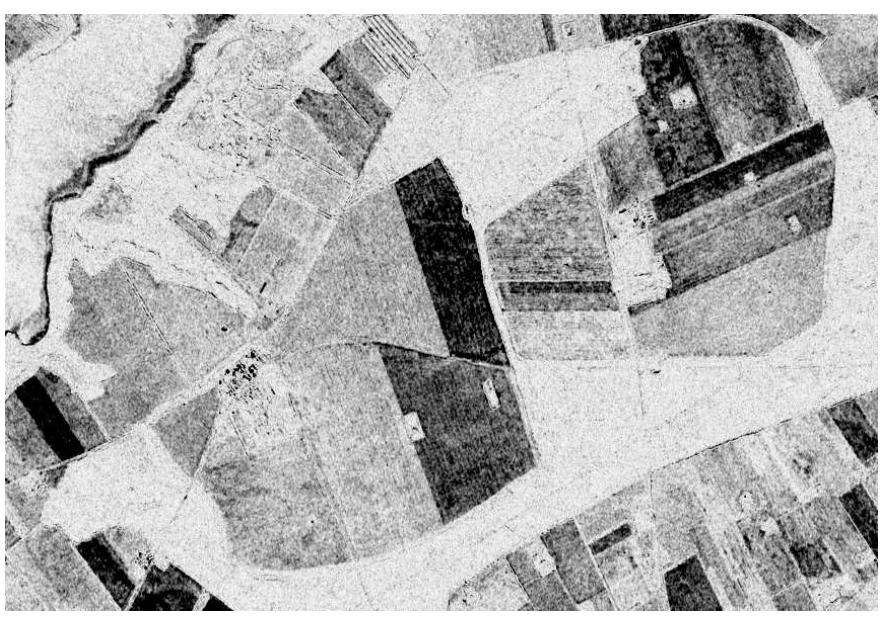

Fig. 4. $L$-band EMISAR change, $\ln Q$, full covariance matrix another with an rms-accuracy of better than one pixel [23].

Figure 3 shows the sum of the diagonal elements (actually $\mathrm{HH}+2 \mathrm{HV}+\mathrm{VV}$ ) in the covariance matrix data for the data acquired on 20 May 1998. This is the so-called span image.

Fields A and B are winter wheat and spring barley, respectively. The winter wheat field has a relatively high phase difference between $\mathrm{HH}$ and $\mathrm{VV}$, probably because of double bounce scattering between the soil surface and the vegetation. The spring barley field has a low phase difference. Fields $C$ and $\mathrm{D}$ are oats and peas fields, respectively. The oats field has a high and the peas field has a low phase difference.

\section{A. Change Detection}

Figure 4 shows $\ln Q$ for the full covariance matrix data stretched linearly between -50 and 0 . Where $Q$ is close to 1 and therefore $\ln Q$ close to 0 which are the bright regions, no or little change occurred between the two points in time. Where $\ln Q$ is small which are the dark regions change did occur.

The bright, very light green areas in Figures 1 and 2 are coniferous forests and we observe very small change for these areas (Figure 4) due to the very stable backscatter from such forest areas (see e.g. [24]). The agricultural fields show different degrees of change, dependent on the crop type. To the extreme left in the image between the lake in the upper left and the coniferous forest three small fields show change, no-change and change behavior, respectively. The field with no change is a beet field, which for both acquisitions is a bare field. The two other fields are spring barley fields, which are bare at the first acquisition and have a $12-14 \mathrm{~cm}$ vegetation layer at the second acquisition. The three large fields (marked $\mathrm{B}$ and A in Figure 3 and the field to the left of field A) in the image are from left to right: grass, winter wheat (A) and spring barley (B). The grass field is virtually unchanged between the two acquisitions. The vegetation height for the winter wheat field (A) has changed from 14 to $42 \mathrm{~cm}$ between the two acquisitions, and we observe a moderate change between the images. It is obvious from the results presented here and the more detailed evaluation in [8] that this change detection method works very well when changes in the polarimetric parameters are experienced.

\section{B. Edge Detection}

The edge detection is performed by visiting each pixel in the image in succession, and for each pixel applying a set of filters with different orientations. The filters estimate the mean covariance matrices on each side of the central pixel, and the test of equality of these two mean covariance matrices determines whether an edge is detected by the current filter or not; see [11], [12] for further details.

Figure 5 shows edges detected using the diagonal elements in the $L$-band EMISAR data from 20 May 1998. Figure 6 shows edges detected using the azimuthal symmetric data from the same date.

The advantage of including the polarimetric information in the edge detection is clearly seen. Especially, the difference is seen for the edges between fields $\mathrm{A}$ and $\mathrm{B}$, and $\mathrm{C}$ and $\mathrm{D}$, respectively (Figure 3 ). In these cases, no edge is detected when only the backscatter coefficients are used in the edge detection (Figure 5), whereas the edge is detected when the polarimetric information is included (Figure 6).

\section{Segmentation}

The MUM (Merge Using Moments) segmentation algorithm for single-channel SAR images was proposed in [25]. As a first step the MUM algorithm severely over-segments the SAR image by dividing it into quadratic segments of size $n \times n$ pixels, where the value of $n$ depends on, e.g., the desired resolution in the resulting segmentation map and on the computational load. Below we use $n=2$. These initial, small segments are then merged based in their statistical properties. The MUM algorithm tries to determine appropriate regions in the image through an iterative region growing process of merging and sorting the segments. The sorting is performed because the order of the merging of the segments affects the final result. It should be noted that the merging performed in 


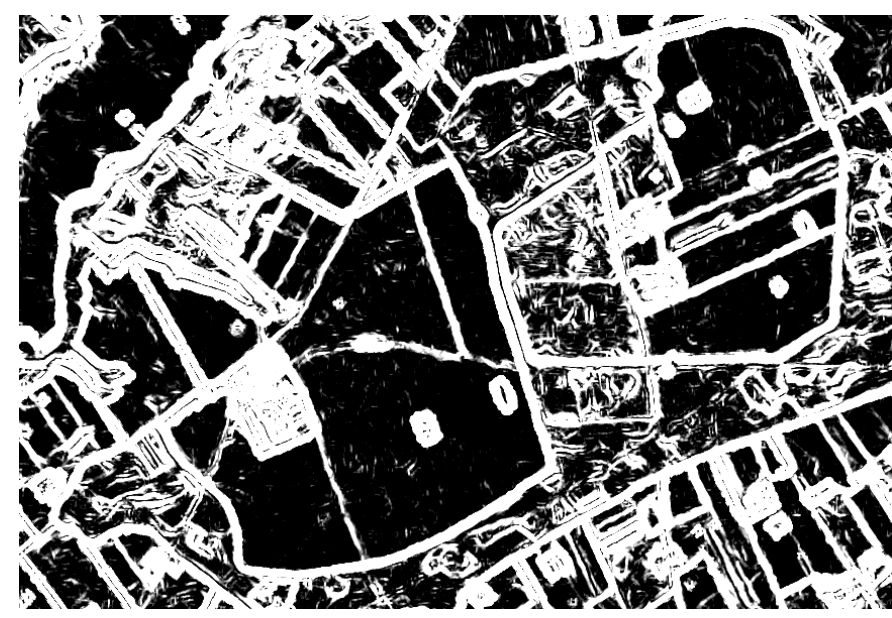

Fig. 5. L-band EMISAR data from 20 May 1998, edges using diagonal elements

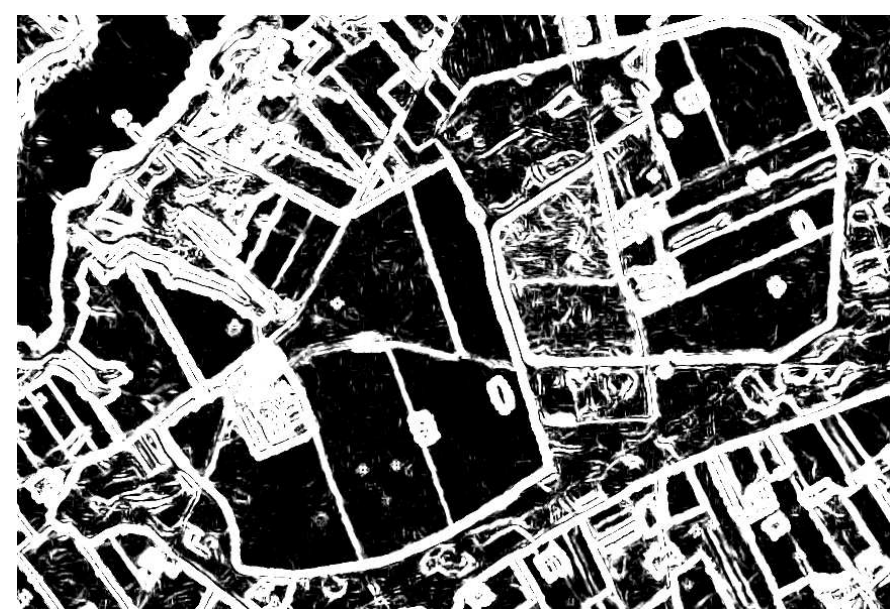

Fig. 6. $\quad L$-band EMISAR data from 20 May 1998, edges using azimuthal symmetry

[25] is based on the Student's t-test, which is only appropriate for Gaussian data, hence an improved segmentation result may be obtained by applying the proper test statistic for Gamma distributed intensities for single-channel SAR images as shown in [26]. Here, the test statistic outlined above is used for segment merging in the multi-look polarimetric data.

Figure 7 shows segmentation using the diagonal elements in the $L$-band EMISAR data from 20 May 1998. Figure 8 shows segmentation using the azimuthal symmetric data from the same date. The colours chosen for the segments are arbitrary.

The advantage of including the polarimetric information in the segmentation algorithm is clearly seen. Especially, the difference is seen for the segments covering fields A and $\mathrm{B}$, and $\mathrm{C}$ and $\mathrm{D}$ (Figure 3). For both cases, the two neighboring fields (i.e. $\mathrm{A}$ and $\mathrm{B}$, and $\mathrm{C}$ and $\mathrm{D}$, respectively) are merged when only the backscatter coefficients are used (Figure 7), whereas they are not merged when the polarimetric information is included (Figure 8).

\section{CONCLUSiOnS AND Future WORK}

A test statistic for the equality of two matrices following the complex Wishart distribution is given with an asymptotic probability for obtaining a smaller value of the test statistic. These measures are applied to change detection, edge detection and segmentation in polarimetric synthetic aperture radar data. The test statistic and the associated probability measure combines the full polarimetric signal in a unified way. As opposed to existing methods that deal with each polarization marginally in turn followed by some heuristic fusion step, this test statistic allows for the full polarimetric information simultaneously.

With the Japanese ALOS in space, the German TerraSAR-X and the Canadian RADARSAT- 2 coming up, methods as the ones described here are expected to become very important for future work on polarimetric data.

Ongoing work includes an object oriented approach in which the Wishart based segmentation is followed by the Wishart based change detection of the segments after allowing for the increased number of equivalent looks of the segments

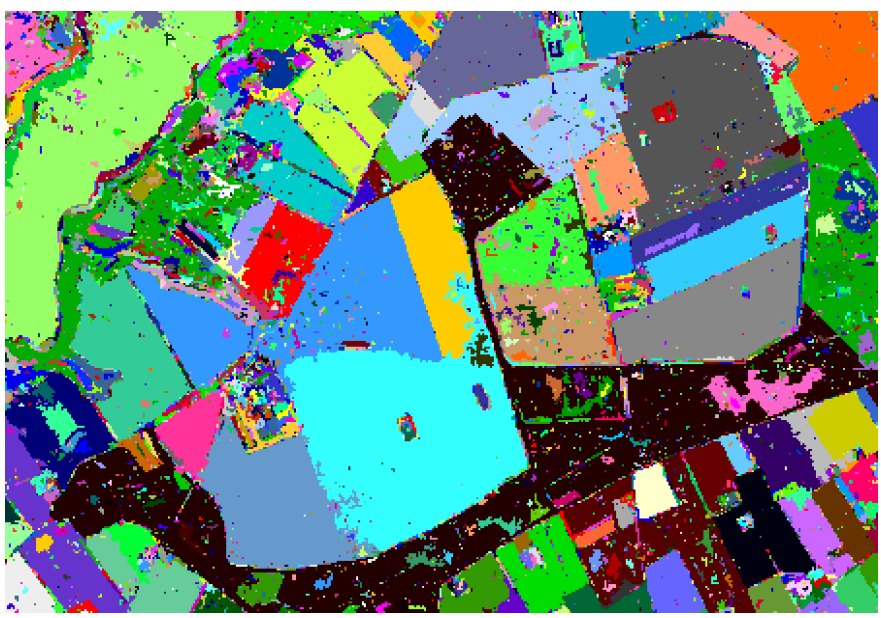

Fig. 7. $L$-band EMISAR data from 20 May 1998, segmentation using diagonal elements

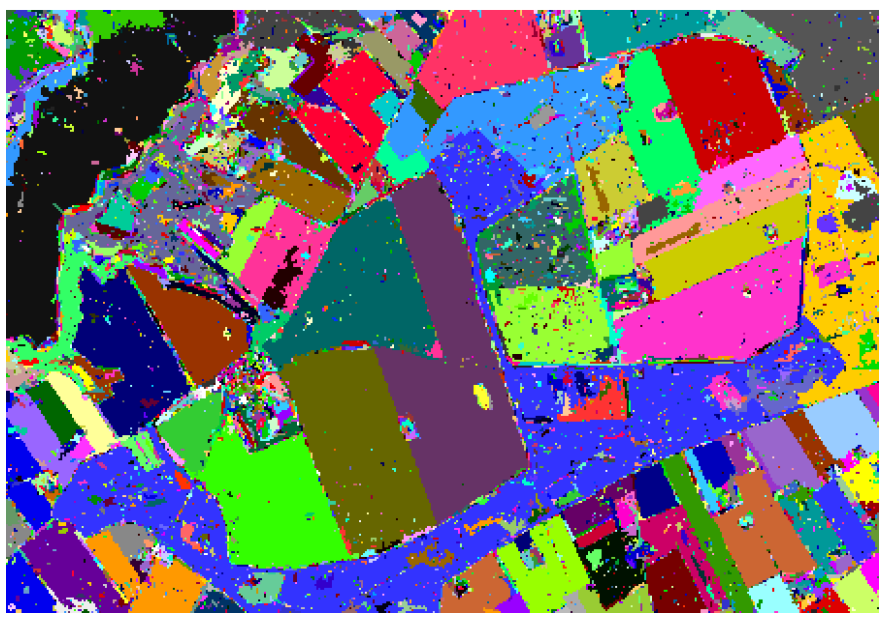

Fig. 8. L-band EMISAR data from 20 May 1998, segmentation using azimuthal symmetry 
[27]. Also, work on the application to low equivalent number of looks with a closer investigation of the asymptotic distribution in Equations 10 and 20 is in progress.

\section{REFERENCES}

[1] F. T. Ulaby, R. K. Moore, and A. K. Fung, Microwave Remote Sensing: Active and Passive, vol. 3, Artech, Dedham, MA, 1986.

[2] H. Skriver, M. T. Svendsen, and A. G. Thomsen, "Multitemporal $L$ and $C$-band polarimetric signatures of crops," IEEE Transactions on Geoscience and Remote Sensing, vol. 37, pp. 2413-2429, 1999.

[3] J. J. van Zyl and F. T. Ulaby, "Scattering matrix representation for simple targets," in Radar Polarimetry for Geoscience Applications, F. T. Ulaby and C. Elachi, Eds. Artech, Norwood, MA, 1990

[4] A. A. Nielsen, R. Larsen, and H. Skriver, "Change detection in bitemporal EMISAR data from Kalø, Denmark, by means of canonical correlations analysis," in Proceedings of the Third International Airborne Remote Sensing Conference and Exhibition, Copenhagen, Denmark, 7-10 July 1997.

[5] A. A. Nielsen, K. Conradsen, and J. J. Simpson, "Multivariate alteration detection (MAD) and MAF post-processing in multispectral, bi-temporal image data: New approaches to change detection studies," Remote Sensing of Environment, vol. 19, pp. 1-19, 1998.

[6] A. A. Nielsen, "Multi-channel remote sensing data and orthogonal transformations for change detection," in Machine Vision and Advanced Image Processing in Remote Sensing, I. Kanellopoulos, G. G. Wilkinson, and T. Moons, Eds., pp. 37-48. Springer, 1999.

[7] A. A. Nielsen, "The regularized iteratively reweighted MAD method for change detection in multi- and hyperspectral data," IEEE Transactions on Image Processing, vol. 16(2), pp. 463-478, 2007.

[8] K. Conradsen, A. A. Nielsen, J. Schou, and H. Skriver, "A test statistic in the complex Wishart distribution and its application to change detection in polarimetric SAR data," IEEE Transactions on Geoscience and Remote Sensing, vol. 41(1), pp. 4-19, 2003.

[9] K. Conradsen, A. A. Nielsen, H. Skriver, and J. Schou, "Change detection in polarimetric SAR data and the complex Wishart distribution," IEEE International Geoscience and Remote Sensing Symposium, IGARSS, Sydney, New South Wales, Australia, 9-13 July 2001.

[10] H. Skriver, A. A. Nielsen, and K. Conradsen, "Evaluation of the Wishart test statistic for polarimetric data," Invited to the IEEE International Geoscience and Remote Sensing Symposium, IGARSS, Toulouse, France, 21-25 July 2003.

[11] J. Schou, H. Skriver, K. Conradsen, and A. A. Nielsen, "CFAR edge detector for polarimetric SAR data," IEEE Transactions on Geoscience and Remote Sensing, vol. 41(1), pp. 20-32, 2003.

[12] H. Skriver, J. Schou, K. Conradsen, and A. A. Nielsen, "Polarimetric edge detector based on the complex Wishart distribution," IEEE International Geoscience and Remote Sensing Symposium, IGARSS, Sydney, New South Wales, Australia, 9-13 July 2001.

[13] J. Schou, Feature Extraction for Topographic Mapping, Ph.D. thesis, Ørsted•DTU, Technical University of Denmark, Lyngby, 2001.
[14] P. Lombardo, M. Sciotti, T. M. Pellizzeri, and M. Meloni, "Optimum model-based segmentation techniques for multifrequency polarimetric SAR images of urban areas," IEEE Transactions on Geoscience and Remote Sensing, vol. 41(9), pp. 1959-1975, 2003.

[15] H. Skriver, J. Schou, A. A. Nielsen, and K. Conradsen, "Polarimetric segmentation using the complex Wishart test statistic," IEEE International Geoscience and Remote Sensing Symposium, IGARSS, Toronto, Ontario, Canada, 24-28 June 2002.

[16] A. A. Nielsen, K. Conradsen, and H. Skriver, "Polarimetric synthetic aperture radar data and the complex Wishart distribution". Proceedings of the 13th Scandinavian Conference on Image Analysis, SCIA 2003, Springer Lecture Notes on Computer Science 2749 pp. 1082-1089. Gothenburg, Sweden, 29 June-2 July 2003.

[17] H. Skriver, J. Schou, and W. Dierking, "Land-Cover Mapping Using Multitemporal, Dual-Frequency Polarimetric SAR data", IEEE International Geoscience and Remote Sensing Symposium, IGARSS, pp. 331$333,2000$.

[18] J. Schou J. and H. Skriver, "Restoration of Polarimetric SAR Images Using Simulated Annealing," IEEE Transactions on Geoscience and Remote Sensing, vol. 39(9), pp. 2005-2016, 2001.

[19] R. Touzi, A. Lopes, and P. Bousquez, "A statistical and geometrical edge detector for SAR images," IEEE Transactions on Geoscience and Remote Sensing, vol. 26, no. 6, pp. 764-773, Nov. 1988.

[20] A. Lopes, E. Nezry, R. Touzi, and H. Laur, "Structure detection and statistical adaptive speckle filtering in SAR images," International Journal of Remote Sensing, vol. 13, no. 9, pp. 1735-1758, 1993.

[21] S. N. Madsen, E. L. Christensen, N. Skou, and J. Dall, "The Danish SAR system: Design and initial tests," IEEE Transactions on Geoscience and Remote Sensing, vol. 29, pp. 417-476, 1991.

[22] E. L. Christensen, N. Skou, J. Dall, K. Woelders, J. H. Jørgensen, J. Granholm, and S. N. Madsen, "EMISAR: An absolutely calibrated polarimetric $L$ - and $C$-band SAR," IEEE Transactions on Geoscience and Remote Sensing, vol. 36, pp. 1852-1865, 1998.

[23] W. Dierking, J. Schou, and H. Skriver, "Change detection of small objects and linear features in multi-temporal polarimetric images," in Proceedings of the International Geoscience and Remote Sensing Symposium (IGARSS), IEEE, Ed., Honolulu, Hawaii, USA, 24-28 July 2000, pp. 1693-1695.

[24] W. Dierking and H. Skriver, "Change detection for thematic mapping by means of airborne multi-temporal polarimetric SAR imagery," IEEE Transactions on Geoscience and Remote Sensing, vol. 40, no. 3, pp. 618-636, 2002

[25] R. Cook, I. McConnell, and C. Oliver, "MUM (Merging Using Moments) segmentation for SAR images," in SPIE vol. 2316, 1994, pp. 92-103.

[26] C. Oliver and S. Quegan, Understanding Synthetic Aperture Radar Images, Artech House, 1998.

[27] H. Skriver, A A. Nielsen, and K. Conradsen, Segment-based change detection for Polarimetric SAR Data. IEEE International Geoscience and Remote Sensing Symposium, IGARSS 2006, Denver, Colorado, USA, 31 July-4 August 2006. 